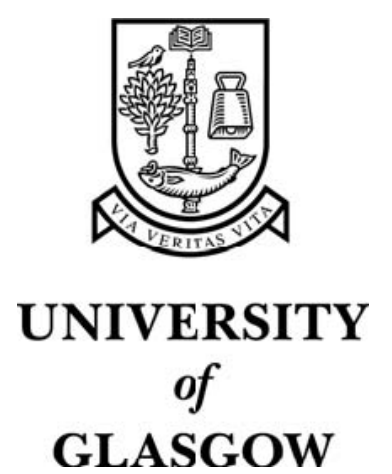

Yassein, M. B. and Ould Khaoua, M. and Mackenzie, L. M. and Papanastasiou, S. (2006) Performance evaluation of adjusted probabilistic broadcasting in MANETs. In, 2nd IEEE International Symposium on Dependable, Autonomic and Secure Computing., 29 September - 1 October 2006, pages pp. 245-249, Indianapolis.

http://eprints.gla.ac.uk/3406/ 


\title{
Performance Evaluation of Adjusted Probabilistic Broadcasting in MANETs
}

\author{
M. Masadeh Bani Yassein, M. Ould Khaoua, L. M. Mackenzie and S. Papanastasiou \\ Department of Computing Science \\ University of Glasgow \\ Glasgow, G12 8RZ, U.K. \\ Email: \{muneer, mohamed, lewis, stelios\}@dcs.gla.ac.uk
}

\begin{abstract}
Appropriate use of a probabilistic broadcasting method in MANETs can decrease the number of rebroadcasts, and as a result reduce the opportunity of contention and collision among neighbouring nodes. In this paper we evaluate the performance of Adjusted Probabilistic flooding by comparing it to "simple" flooding as used with the Ad Hoc on Demand Distance Vector (AODV) routing protocol as well as a fixed probabilistic approach. The results reveal that the Adjusted Probabilistic flooding exhibits superior performance in terms of both reachability and saved rebroadcasts.
\end{abstract}

\section{Introduction}

The distributed, wireless, and self-configuring nature of Mobile Ad Hoc Networks (MANETs) make them suitable for a wide variety of applications, including military operations, rescue and law enforcement missions as well as disaster recovery scenarios $[1,2]$. In the Ad Hoc on Demand Distance Vector (AODV) routing protocol for MANETs, whenever a source $\mathrm{S}$ needs to communicate with a destination $\mathrm{D}$, it checks for an existing route to $\mathrm{D}$ in its table. If the route is not present, it initiates a route discovery by broadcasting a RREQ (Route Request) packet which is flooded [6] into the network in a controlled manner, until it reaches the destination or until it reaches a node, which knows a route to the destination. Then, the destination or intermediate node sends back a Route Reply (RREP) message, which includes the number of hops in between. Each node receiving the RREP message records a forward route to the destination and, thus, knows only the next hop required for a given route and, not the complete

One of the earliest broadcast mechanisms proposed in the literature is simple or "blind" flooding [3] where each node receives and then re-transmits the message to all its neighbours. The only 'optimisation' applied to this technique is that nodes may remember broadcast messages received and do not act if they receive repeated copies of the same message [7]. However, a straightforward flooding broadcast is usually costly and results in serious redundancy and collisions in the network; such a scenario has often been referred to as the broadcast storm problem [3], and has generated many challenging research issues.

A probabilistic approach to flooding has been suggested in $[5,6,7]$ as a means of reducing redundant rebroadcasts and alleviating the broadcast storm problem. In the probabilistic scheme, when receiving a broadcast message for the first time, a node rebroadcasts the message with a pre-determined probability $\mathrm{p}$; every node has the same probability to rebroadcast the message. When the probability is $100 \%$, this scheme reduces to simple flooding. Studies [3] have shown that probabilistic broadcasts incur significantly lower overhead compared to blind flooding while maintaining a high degree of propagation for the broadcast messages. This paper focuses on evaluating the performance of our Adjusted Probabilistic flooding scheme by comparing it with the AODV protocol simple flooding technique as well as a fixed probabilistic approach. The relative merits or characteristics of each method are discussed in turn and subsequent observations offer insight on possible applications of each.

The rest of this paper is organized as follows: In Section 2, we introduce the related work of broadcasting in MANETs. In Section 3, we describe our dynamic probabilistic approach, highlighting the difference in our approach from other similar approaches, and analyze relative strength and weakness. In Section 4, we evaluate our approach and present the simulation results. Section 5, concludes the paper and offers suggestions for future work.

\section{RELATED WORK}

One of the earliest broadcast mechanisms is flooding, where every node in the network retransmits a message to its neighbours upon receiving it for the first time. Although flooding is very simple and easy to implement, it can be very costly and may lead to a serious problem, often known as the broadcast storm problem [3] that is characterized by high redundant packet retransmissions, network contention and 
collision. Ni et. al. [4] have studied the flooding protocol analytically and experimentally. Their obtained results have indicated that rebroadcasts could provide at most $61 \%$ additional coverage and only $41 \%$ additional coverage on average over that already covered by previous transmissions. Therefore, rebroadcasts are very costly and should be used with caution.

In [3], Williams et al. have classified the broadcasting techniques into the following four categories: simple flooding, probability-based, area-based, and neighbour knowledge scheme. In the flooding scheme, every node retransmits its neighbours as a response to every newly received packet. The probability-based scheme is a simple way of controlling message floods. Each node rebroadcasts with a predefined probability $p$ [5]. Obviously when $p=1$ this scheme resembles simple (blind) flooding. In the area based scheme, a node determines whether to rebroadcast a packet or not by calculating and using its additional coverage area. Of these, of interest in this study is the probabilistic scheme family of variants. In this category of broadcasting techniques, a mobile node rebroadcasts packets according to a certain probability.

Cartigny and Simplot [6] have described a probabilistic scheme but the probability $p$ of a node retransmitting a message is computed from the local density $n$ (i.e. the number of neighbours) and a fixed value $\mathrm{k}$ for the efficiency parameter to achieve the reachability of the broadcast. This model [6] has the disadvantage of being locally uniform. Indeed, each node of a given area receives a broadcast and determines the probability according to a constant efficiency parameter to achieve the reachability and from the local density [6].

Zhang and Dharma [7] have described a dynamic probabilistic scheme. They use a combination of probabilistic and counterbased approaches. The value of a packet counter does not necessarily correspond to the exact number of neighbors from the current host, since some of its neighbours may have suppressed their rebroadcasts according to their local rebroadcast probability. On the other hand, the decision to rebroadcast is made after a random delay, which increases latency.

A format sheet with the margins and placement guides is available in both Word and PDF files as <format.doc $>$ and $<$ format.pdf $>$. It contains lines and boxes showing the margins and print areas. If you hold it and your printed page up to the light, you can easily check your margins to see if your print area fits within the space allowed.

\section{PROBABILISTIC FLOODING}

The probabilistic scheme [5] is one of the alternative approaches to simple flooding that aims to reduce redundancy through rebroadcast timing control in an attempt to alleviate the broadcast storm problem. In this scheme, when receiving a broadcast message for the first time, a node rebroadcasts the message with a pre-determined probability $p$ so that every node has the same probability to rebroadcast the message, regardless of its number of neighbours.
In dense networks, multiple nodes share similar transmission range. Therefore, these probabilities control the frequency of rebroadcasts and thus might save network resources without affecting delivery ratios. Note that in sparse networks there is much less shared coverage; thus some nodes will not receive all the broadcast packets unless the probability parameter is high. So if the rebroadcast probability $p$ is set to a far smaller value, reachability will be poor. On the other hand, if $p$ is set far large, many redundant rebroadcasts will be generated. The need for dynamic adjustment, thus, rises.

The rebroadcast probability should be set high at the hosts in sparser areas and low at the hosts in denser areas. Our simple method for density estimation requires mobile hosts to periodically exchange HELLO messages between neighbours to construct a 1-hop neighbour list at each host. A high a number of neighbours implies that the hosts in denser areas, a low number of neighbors imply that the host is in sparser areas. We increase the rebroadcast probability if the value of the number of neighbours is too low (or similarly if the current node is located in a sparse neighbourhood), which indirectly causes the probability at neighbouring hosts to be incremented. Similarly, we decrease the rebroadcast probabilities if the value of number of neighbours is too high.

This kind of adaptation causes a dynamic stability between rebroadcast probabilities number of neighbours values among neighbouring hosts. Intuitively, the probabilities at the stability states should lead to optimal solutions. We adopt a simple adaptation algorithm. A brief outline of the adjusted probabilistic flooding algorithm is presented in Figure 1 and operates as follows. On hearing a broadcast message $m$ at node $X$, the node rebroadcast a message according to a high probability if the message is received for the first time, and the number of neighbours of node $X$ is less than average number of neighbours typical of its surrounding environment. Hence, if node $X$ has a low degree (in terms of the number of neighbours), retransmission should be likely. Otherwise, if $X$ has a high degree its rebroadcast probability is set low.

The adjusted rebroadcast probability for probabilistic broadcasting algorithm for each node is briefly presented below.

\section{The Adjusted Probabilistic Flooding Algorithm}

\section{Protocol receiving () \\ On hearing a broadcast packet $m$ at node $X$ \\ Get the Broadcast ID from the message; $\bar{n}$ average number of neighbor (threshold value);}

Get degree $n$ of a node X (number of neighbors);

$$
\begin{aligned}
& \text { If packet } m \text { received for the first time then } \\
& \qquad \begin{array}{l}
\text { If } n<\bar{n} \text { then } \\
\text { Node } X \text { has a low degree: } \\
\text { set high rebroadcast probability } p=p_{1} ;
\end{array}
\end{aligned}
$$$$
\text { Else } n \geq \bar{n}
$$$$
\text { Node X has a high degree: }
$$ 


\section{set low rebroadcast probability $p=p_{2}$; End if \\ End if}

Generate a random number $R N$ over $[0,1]$

If $R N \leq p$ rebroadcast message; otherwise, drop.

Figure 1: An outline of the adjusted probabilistic flooding algorithm.

Our algorithm is a combination of probabilistic and knowledge based approaches. It dynamically adjusts the rebroadcast probability $p$ at each mobile host according to the value of the local number of neighbours. The value of $p$ changes when the host moves to a different neighbourhood. In a sparser area, the rebroadcast probability is larger and in denser area, the probability is lower. Compared with the probabilistic approach where $p$ is fixed, our algorithm achieves higher saved rebroadcast. On the other hand, the decision to rebroadcast is made immediately after receiving a packet in our algorithm without any delay.

We present an estimate of average neighbour number as the basis for the selection of the value of $p$. Let $A$ be the area of an ad hoc network, $N$ be the number of mobile hosts in the network, and $R$ be the transmutation range. The average number of neighbour $\bar{n}$ can be obtained as shown below.

\section{Equation 1:Average Number of neighbours}

$$
\bar{n}=(N-1) 0.8 \frac{\pi^{2}}{A}
$$

\section{PERFORMANCE EVALUATION}

In this section, we evaluate the performance of adjusted probability, probabilistic algorithms with fixed rebroadcast probability. We have implement all three algorithms in the AODV protocol. The metrics for comparison include the average number of routing request rebroadcasts, reachability, the average number of collisions, end-to-end delay, and throughput.

We have used the ns-2 simulator (v.2.27) to conduct extensive experiments to evaluate the performance of probabilistic flooding. The original AODV protocol uses simple flflooding to broadcast routing requests. We have implemented two AODV variations: one using probabilistic method with Fixed Probability (FP-AODV), and the other using our Adjusted Probabilistic algorithm (AP-AODV). The main idea of APAODV and FP-AODV is to reduce the no. of rebroadcasts during route discovery, thereby reducing the network traffic and decrease the probability of channel contention and packet collision.

Since our algorithm is based on a probabilistic approach it does not fit every scenario, as there is a small chance that the route requests cannot reach the destination. We have to generate the route request again if the previous route request failed to reach the destination. The AODV protocol, on the contrary, uses flooding in the route discovery phase, which means that all route requests will reach their destinations if the network is not partitioned. Based on this observation, our algorithm should perform better than AODV in dense networks.

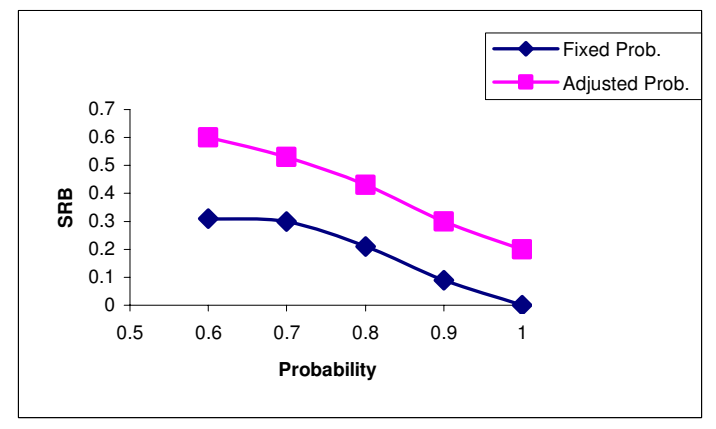

Figure 2: The SRB vs. the rebroadcast probability with node speed $10 \mathrm{~m} / \mathrm{s}$.

The network considered for the performance analysis of the rebroadcast probability vs density varies from 25 to 100 nodes placed randomly on $600 \times 600 \mathrm{~m}$ area, with each node engaging in communication transmitting within 250 meter radius and having bandwidth of $2 \mathrm{Mbps}$. The random waypoint model is used to simulate 25 mobility patterns with retransmission probabilities ranging from 0.5 to 1.0 percent with 0.1 percent increment per trial.

In short, the random waypoint model considers nodes that follow a motion-pause recurring mobility state. Each node at the beginning of the simulation remains stationary for pause time seconds, then chooses a random destination and starts moving towards it with speed selected from a uniform distribution $(0$, max_speed]. After the node reaches that destination, it again stands still for a pause time interval and picks up a new destination and speed. This cycle repeats until the simulation terminates. The maximum speeds of $1,5,10,20 \mathrm{~m} / \mathrm{sec}$ and pause times of 0 seconds are considered for the purposes of this study. The simulation parameters are summarised in Table 1 below.

Table 1: The parameters used in the simulation.

\begin{tabular}{|l|l|}
\hline Parameter & Value \\
\hline Transmitter range & 250 meter \\
\hline Bandwidth & 2Mbit \\
\hline Interface queue length & 50 packets \\
\hline Simulation time & 900 seconds \\
\hline Pause time & 0 \\
\hline Packet size & 512 bytes \\
\hline Topology size & $600 \times 600$ meter $^{2}$ \\
\hline Number of node & $25,50,75,100$ \\
\hline Maximum speed & $1,5,1020 \mathrm{~m} / \mathrm{sec}$ \\
\hline
\end{tabular}

The performance of broadcast protocols can be measured by a variety of metrics $[1,3,4]$ A commonly used metric is the number of message re-transmissions with respect to the number 
of nodes in the network [3]. In this work, we use rebroadcast savings, which is a complementary measure and is precisely defined below. The next important metric is reachability, which is defined in terms of the ratio of nodes that received the broadcast message out of all the nodes in the network. The formal definitions of these two metrics are given as follows [4].

Saved ReBroadcasts (SRB): Let $r$ be the number of nodes that received the broadcast message and let and $t$ be the number of nodes that actually transmitted the message. Saved rebroadcast is then defined by $(r-t) / r$.

Reachability (RE): is the percentage of nodes that received the broadcast message to the total number of nodes in the network. For meaningful information, the total number of nodes should include those nodes that are part of a connected component in the network. For disconnected networks this measure should be applied to each of the components separately.

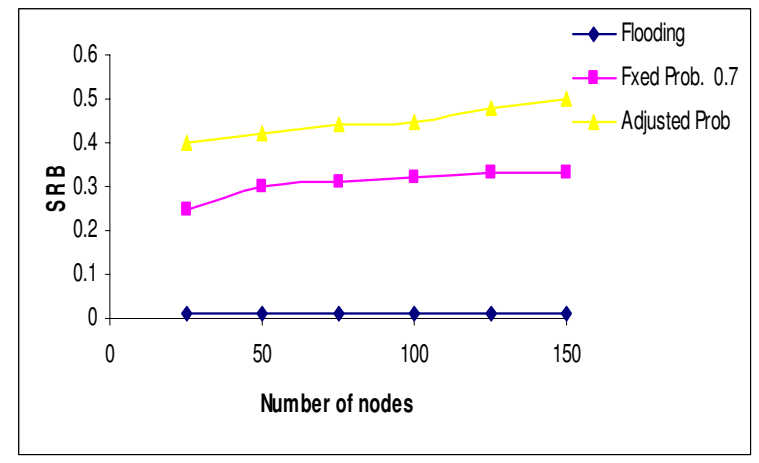

Figure 3: SRB of three broadcast schemes against network density with node speed $10 \mathrm{~m} / \mathrm{s}$.

We have compared the saved broadcast (SRB) in fixed probability and our algorithm Adjusted Probabilistic. Figure 2 shows our algorithm can significantly reduce the number of saved rebroadcasts (SRB) with rebroadcast probabilities ranging from 0.5 to 1.0 percent with 0.1 percent increment per trial for a network with 50 nodes and maximum speed $20 \mathrm{~m} / \mathrm{s}$ and 0 pause time. Figure 3 shows the saved rebroadcast (SRB) of the fixed probabilistic and our Adjusted Probabilistic Algorithm.



Figure 4: Reachability of three broadcast algorithms.

The SRB of Adjusted Probabilistic is $40 \%$ in low-density networks (25 nodes) and 50\% in high-density networks (150 nodes). The SRB of the fixed probabilistic scheme with the probability assigned to 0.7 in any density of network is around $30 \%$. Figure 4 shows that reachability increases when network density increases, regardless of what kind of the algorithm is used. The simple flooding method has the best performance in reachability, as expected. The performance of adjusted probabilistic algorithm shows that the reachability is above $95 \%$ in any density of the network. In all network densities, the reachability of our algorithm performs better than the probabilistic scheme when probability Is set to 0.7 . In higher density networks, i.e., 120 hosts and above, the reachability of our approach and flooding are evenly matched, with both performing very adequately (close to $100 \%$ ).

\section{CONCLUSIONS}

This paper has weigh up the performance of Adjusted Probabilistic flooding on the AODV protocol which is based on simple flooding in MANETs to increase saved rebroadcasts. The algorithm determines the rebroadcast probability by taking into account the network density. In order to improve the saved rebroadcasts, the rebroadcast probability of low density nodes is increased while that of high density nodes is decreased. Compared with the simple flooding, our simulation results have shown that the adjusted probabilistic flooding algorithm can improve the saved broadcast up to $50 \%$ without scanting reachability, even under conditions of high mobility and density.

As a prospect for future work, we plan to evaluate the performance of Adjusted Probabilistic flooding on the Dynamic Source Routing (DSR) algorithm. Then we aim to build an analytic model for our approach in order to facilitate the exploration of the optimal adaptation strategy, with regard to probability setting and network density. Finally, since the technique avails itself to various types of network-wide dissemination, we plan to integrate it with a proactive routing protocol, namely OLSR. This protocol already incorporate techniques which reduce the effect of flooding and are orthogonal to our scheme, which implies the opportunity to 
examine if cumulative improvements with our method are possible.

\section{References}

[1] W. Peng, X.C. Lu, on the reduction of broadcast redundancy in mobile ad hoc networks, in Proceedings of the First Annual Workshop on Mobile Ad Hoc Networking \& Computing (MobiHOC, 2000), Boston, Massachusetts, USA, pp. 129-130, 2000.

[2] C-K. Toh, Ad Hoc Mobile Wireless Networks: Protocols and Systems (Prentice-Hall, New York, 2002).

[3] B. Williams, T. Camp, Comparison of broadcasting techniques for mobile ad hoc networks. (MOBIHOC 2002), pp. 194-205, 2002.

[4] S.-Y. Ni, Y.-C. Tseng, Y.-S. Chen, J.-P. Sheu, The broadcast storm problem in a mobile ad hoc network, Wireless Networks, vol. 8, no. 2, pp.153-167, 2002

[5] Y. Sasson, D. Cavin, A. Schiper, Probabilistic broadcast for flooding in wireless mobile ad hoc networks, Technical report IC/2002/54, EPFL, 2002.

[6] J. Cartigny, D. Simplot. Border node retransmission based probabilistic broadcast protocols in ad-hoc networks, Telecommunication Systems, vol. 22, no 1-4, pp. 189-204, 2003.

[7] Q. Zhang, D.P. Agrawal , Dynamic probabilistic broadcasting in MANETs, Journal of Parallel Distributed Computing Vol 65, pp. 220-233, 2005. 